there are several records of these two properties for di-n-butyl ether and $n$-butyl propionate, for both of which density at $0^{\circ} \mathrm{C}$. alone is given, that for the latter compound dating from 1886. The preferred. melting point for $m$-hydroxybenzaldehyde is evidently that of Jowett (1900), who later identified his product as $p$-hydroxyacetophenone; there is ample corroboration of the bracketed value.

Generous use of cross-references avoids duplication of entries with corresponding danger of discrepancies. This has, however, occurred with some of the sulpha drugs and their acetyl derivatives; some of these are also listed as the $p$-aminobenzenesulphonyl derivatives of the heterocyclic amines with melting points which differ by up to $8 \mathrm{deg}$. C.

A particular teature is the inclusion of a large number of organic compounds of natural origin; but asarinin, stevioside (of interest for its sweet taste) and dihydronorguaiaretic acid (NDGA) are three such products which appear to have escaped the net. Ditert-amyl peroxide is listed (under A, contrary to the declared naming of 'double' derivatives) but not the commercially important tert-butyl compound.

These various observations must not be taken to imply that the "Dictionary" is bristling with inaccuracies and omissions. They merely show that the editors of books of this kind can never sit back with the comfortable feeling that their work is completely satisfactory ; there is always some dusting and polishing to be done. The reviewer will continue to use the "Dictionary", as a first source of information about compounds, except those newly described, with the assurance that, more frequently than not, the search will not be in vain.

B. A. Ellis

\section{LINEAR OPERATORS}

Linear Analysis

Measure and Integral, Banach and Hilbert Space, Linear Integral Equations. By Prof. Adriaan Cornelis Zaanen. (Bibliotheca Mathematica : a Series of Monographs on Pure and Applied Mathematics. Volume 2.) Pp. vii +602. (Amsterdam: NorthHolland Publishing Company; New York: Interscience Publishers, Inc., 1953.) 38 florins ; $76 s$.

\section{Linear Operators}

Spectral Theory and Some Other Applications. By Dr. Richard G. Cooke. Pp. xii +454. (London : Macmillan and Co., Ltd.; New York : St. Martin's Press, Inc., 1953.) 52s. 6d. net.

$\mathrm{T}$ HE general theory of linear operators is mainly a creation of the present century; it originated in the work of Hilbert and Riesz on the theory of integral equations and its extensions, and assumed its modern abstract form in the 1920's in the hands of Banach, von Neumann and Stone. The past twenty-five years have witnessed its growth into the wide-ranging subject known to-day as functional analysis, which may be described as the application of the methods of topological algebra to problems in the theory of functions.

Each of these two books is concerned with certain aspects of this now enormous field. They have a small amount of basic material in common; but their aims are very different. Prof. A. C. Zaanen is mainly interested in topics related to the classical theory of linear integral equations, whereas Dr. R. G. Cooke's principal aim is to acquaint his readers with the spectral theory of self-adjoint linear operators in Hilbert space.

Prof. Zaanen's book begins with an account of the Lebesgue integral on a general measure space, and continues with a detailed discussion, not only of the $L_{p}$ spaces but also of the less familiar Orlicz spaces. We next find a description of the structure of Banach spaces and Hilbert space, and an outline of the theory of bounded linear operators, including the BanachSteinhaus theorem and the closed graph theorem. In order to avoid using the axiom of choice, Prof. Zaanen does not prove the Hahn-Banach theorem on the existence of linear functionals in its most general form, but he shows that it can often be dispensed with; for example, the whole Riesz-Schauder theory of compact (or completely continuous) linear operators is obtained without appealing to it. The Dunford spectral theory is outlined; but there is no account of the spectral theory of general self-adjoint operators in Hilbert space. The applications of the Riesz-Schauder theory to integral equations receive very thorough treatment, the Fredholm theory is extended to a wider class of integral operators than usual (those that are completely of finite double norm), and a very detailed discussion is given of various classes of symmetrizable integral operators.

The book is too long and detailed to be suitable as a first introduction to the subject; but it will be an indispensable work of reference for these aspects of functional analysis. Particularly welcome are the numerous illustrative examples, to most of which are attached either hints for solution or references to the original literature.

Dr. Cooke's book is a successor to his "Infinite Matrices and Sequence Spaces", published in 1950. The first five chapters contain an introduction to Hilbert space and its applications in quantum mechanies, and a very full account of the spectral theorem for hypermaximal (or self-adjoint) linear operators, both bounded and unbounded. A number of the known proofs of the theorem are given in detail. The remaining two chapters deal with topics having little direct connexion with the first part of the book. Chapter 6 , on projective convergence in matrix spaces and rings, is a pendant to the author's earlier book, and describes some recent investigations on linear operators in sequence spaces. Chapter 7 is on Banach algebras ; it contains a useful account of the Gelfand representation of a commutative Banach algebra by functions on the space of maximal ideals, and its applications to the proof of Wiener's theorem on the closure of translations and his Tauberian theorem. Other topics included are the spectacular and powerful Weierstrass-Stone theorem on uniform approximation to continuous functions, the theory of Radon measures, defined as linear functionals on a class of continuous functions, and an application of the theory of Banach algebras with an involution to the proof of Bochner's theorem on positive definite functions.

Much of the material in this book has hitherto been scattered in the periodical literature, some of it being available only in Russian, and the author has performed a useful service in collecting it together. Dr. Cooke writes as an analyst for analysts; the emphasis throughout is on analytic techniques and applications, and the algebraic structures tend to remain in the background.
F. Smithies 